\title{
Silicon photonics wavelength converter based on inter-modal four wave mixing Bragg scattering
}

\author{
C. Lacava*, M.A. Ettabib*, G. Sharp ${ }^{\dagger}$, Y. Jung*, P. Petropoulos*, D.J. Richardson* \\ M. Sorel ${ }^{\dagger}$, F. Parmigiani* \\ *Optoelectronics Research Centre, University of Southampton, Southampton, SO17 1BJ, UK. Email: C.Lacava@ soton.ac.uk \\ ${ }^{\dagger}$ University of Glasgow, Glasgow, G12 8LP, UK
}

\begin{abstract}
We present the first ever inter-modal silicon photonic wavelength converter operated with a telecom-compatible dual-pump CW scheme. We achieve phase-matched inter-modal four-wave-mixing, allowing for wavelength conversion with a pumps-to-signal wavelength detuning of $70 \mathrm{~nm}$.
\end{abstract}

\section{INTRODUCTION}

Nonlinear silicon photonic devices have attracted considerable attention thanks to their ability to show large thirdorder nonlinear effects at moderate pump powers, allowing for advanced on-chip all-optical signal processing functionalities [1]. Optical wavelength conversion is typically realized exploiting four wave mixing (FWM), where the input signal information is transferred to the desired wavelength via a nonlinear process, which requires phase matching among the interacting waves. This is achieved by tailoring the waveguide geometry to engineer the group velocity dispersion and, while it is very effective, it imposes stringent trade-offs in single mode engineered waveguide designs, particularly if extremely broad and wide-band systems are simultaneously required [2]. Multi-mode nonlinear waveguides, possessing the extra degree of freedom given by the capability to carefully excite and control higher order modes, offer more ways to fulfill the required phase matching, in principle allowing for broadband operation at multiple discrete spectral bands, even at spectral locations far away from the pump wavelength(s). For this reason, inter-modal FWM processes in optical fibre have recently regained interest, primarily driven and supported by developments in space division multiplexing communications and technology [3], [4]. A preliminary demonstration of intermodal FWM in a multi mode silicon waveguide was recently reported in [5], where FWM between different modes was observed using a high peak power ps-pulse pump source. In this work, we show the realization of a novel wavelength conversion scheme for telecom settings. We employ, for the first time, inter-modal FWM in a dispersion engineered multimode silicon waveguide, using two $\mathrm{CW}$ pumps, which are positioned outside the signal/idler band. We launch the two CW pump sources in one spatial mode and the signal, detuned by about $70 \mathrm{~nm}$ from the pump wavelengths, in another mode and demonstrate the generation of new signals (idlers) in the same spatial mode as the signal. This demonstration represents a critical step toward the achievement of on-chip tunable discrete wavelength converters for telecommunication applications that can be detuned by hundreds of $\mathrm{nm}$ from the signal/pump wavelengths by exciting phase matched and dispersion tailored modes of a single multi-mode nonlinear waveguide.

\section{OPERATION PRINCIPLE AND EXPERIMENTAL SET-UP}

The wavelength converter was based on a 1-cm long silicon on insulator (SOI) multi-mode waveguide, with a cross section of $5 \times 0.22 \mu \mathrm{m}$. The sample was fabricated using e-beam lithography followed by a single dry etching step. Sample cleaving was required to provide good quality waveguide-access facets. According to our simulations, the fabricated waveguide supports up to 10 modes in the TE-polarization. The operational principle of the wavelength converter is illustrated in Fig. I (a) [2]. The figure shows the simulated group index curves of the first four supported modes as a function of wavelength propagating in our multi-mode waveguide. Inter-modal phase matching is achieved when the propagation constants of the interacting waves satisfy conservation of momentum (as well as conservation of energy). This occurs when the value of the group index of the various modes evaluated at the average wavelength of the waves in the same mode (pump-pump and signal-idler pairs in this instance) are equal [2], [3], see dashed black line in Fig. I (a). These numerical results show the possibility to achieve phase matching across multiple discrete wavelength bands, from $1.55 \mu \mathrm{m}$ up to $2 \mu \mathrm{m}$, by exciting the corresponding high order modes. The experimental set-up is shown in Fig. I (b). Two CW pump signals were coupled at the input of an erbium doped fiber amplifier (EDFA) to achieve the desired level of power. An L-band signal was generated using a tunable $\mathrm{CW}$ laser and then amplified. These single-mode (SM) signals were then coupled to two separate fiber collimators. In order to generate the higher order mode, LP11 mode, from the LP01 spatial mode a bulk optic phase plate (PP) was placed after one of the two launching fiber collimators. Polarization controllers (PCs) were used to guarantee that all the signals were co-polarized relative to each other and aligned with the TE polarization of the waveguide. The two pumps (in the LP01 mode) were coupled with the L-band signal (in the LP11 mode) using a free space beam splitter. The optical beam was coupled to the waveguide by using a 40x objective, by means of the end-fire coupling technique. The LP01 mode excited the TE waveguide fundamental mode (TE00), while the LP11 mode excited the TE10 waveguide mode, see corresponding inset images of Fig. I (b) for their spatial mode distributions after propagation in the waveguide. The beams at the output of the waveguide were coupled back to a SM fiber using a 
(a)

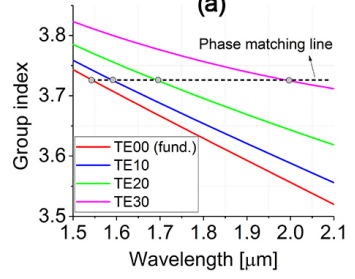

(b)

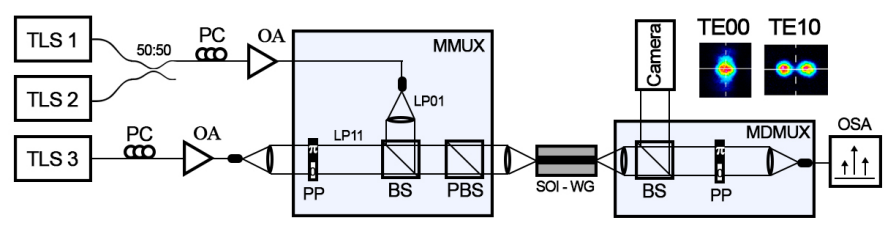

(c)

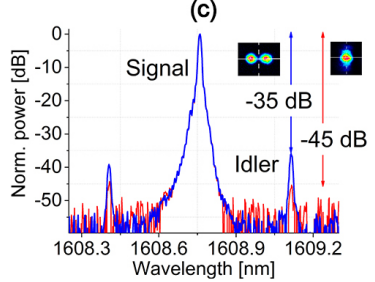

Fig. 1. (a) numerical simulations showing the calculated group index as a function of wavelength for each mode (up to TE30) supported by the waveguide under test; (b) Experimental set-up; (c) Examples of typical spectra at the output sample after inter- (blue curve) and intra- (red curve) modal FWM processes when the signal wavelength is of $1608.67 \mathrm{~nm}$.

second 40x objective and a mode de-multiplexer. In order to de-multiplex the two spatial modes (TE00 and TE10) a second bulk optic PP was placed before the output SM-fiber. The de-multiplexer allowed extinction of either the TE00 or the TE10 optical mode, with a measured modal purity of, at least, $10 \mathrm{~dB}$. Output optical spectra were recorded using an optical spectrum analyzer (OSA). Note that to measure intra-modal FWM we removed the PPs at the input and output of the setup to guarantee that all the signal waves were exciting only the fundamental mode of the waveguide which was then coupled into the SM output fiber.

\section{RESULTS AND DISCUSSION}

We first characterized the linear and nonlinear behavior of our SOI sample. Propagation losses were measured to be 1.5 $\mathrm{dB} / \mathrm{cm}$ for both the TE00 and TE10 modes. We assessed a coupling loss of $7.6 \mathrm{~dB} /$ facet and $8.1 \mathrm{~dB} /$ facet for the TE00 and TE10 modes, respectively. The waveguide nonlinear parameter (for the TE00 mode) was measured to be $8 \mathrm{Wm}^{-1}$. We operated the wavelength converter using the TE00 and TE10 modes (exhibiting an inter-modal nonlinear modal overlap of about $70 \%$ ). According to our numerical simulations phase matching can occur between signals exciting the TE00 mode placed at a wavelength of about $1545 \mathrm{~nm}$ and the TE10 mode placed at a wavelength of about $1610 \mathrm{~nm}$. We placed the two pump signals at wavelengths of $\lambda_{p 1}=1544.75 \mathrm{~nm}$ and $\lambda_{p 2}=1545.25 \mathrm{~nm}$, respectively, while the signal was positioned at $\lambda_{s}=1608.7 \mathrm{~nm}$. In Fig. I (c) we show an example of the signal and the generated idler spectra when a pump power of $120 \mathrm{~mW}$ was coupled into the waveguide both for the intra- and inter- modal FWM processes, respectively. The measured inter-modal FWM efficiency was -35 dB (blue line). For comparison, the intra-modal generated idler was also measured (red line), revealing a conversion efficiency of -45 $\mathrm{dB}$. This $10 \mathrm{~dB}$ improved conversion efficiency demonstrates that phase matching is achieved for the inter-modal process when a pumps-to-signal detuning of $70 \mathrm{~nm}$ was used, while only partial phase matching was observed for the intra-modal process. A second set of measurements was performed in order to assess the bandwidth of the proposed wavelength conversion scheme. We measured the normalized conversion efficiency as a function of the signal wavelength, when the two pump beams were kept fixed at $\lambda_{p 1}=1544.75 \mathrm{~nm}$ and $\lambda_{p 2}=1545.25$ $\mathrm{nm}$, for both the inter and intra-modal FWM processes. In the inter-modal configuration, the phase matching condition was no longer fully achieved when the signal was detuned from the starting wavelength of $\lambda_{s}=1608.7 \mathrm{~nm}$, and as a consequence we observed a drop in terms of conversion efficiency ( $3 \mathrm{~dB}$ bandwidth of $14 \mathrm{~nm}$ ). This measurement was also performed to characterize the intra-modal FWM configuration. In this case, the FWM efficiency is relatively constant as a function of signal wavelength due to the short length of the sample (i.e. showing low net-dispersion), with a small increase of efficiency as the signal is tuned closer to the pump wavelengths as the process become better phase matched. The inter-modal phase matching bandwidth was also assessed by measuring the FWM-efficiency as a function of the signal to idler detuning, exhibiting a $3 \mathrm{~dB}$ bandwidth of $11 \mathrm{~nm}$ for the red-shifted FWM BS generated idlers.

\section{CONCLUSION}

We demonstrated, for the first time, a dual- pump CW silicon photonics wavelength converter, using an inter-modal FWM BS process. We report a conversion efficiency of -35 $\mathrm{dB}$, between the TE00 and the TE10 waveguide modes, when a pump power of $120 \mathrm{~mW}$ is coupled into the waveguide, with a pumps-to-signal detuning of $70 \mathrm{~nm}$. Higher conversion efficiency could be achieved using longer waveguides, or higher pump power levels in combination with two photon absorption-free materials (such as $\mathrm{SiN}$ ). Our simulated group index curves predict multiple phase matching bands, reaching $2 \mu \mathrm{m}$, if the signal/pumps are used to excite higher order modes of the same multi-mode waveguide.

\section{ACKNOWLEDGMENT}

This work was supported by the Engineering and Physical Sciences Research Council (EPSRC) through the grant EP/P026575/1 and the Project Silicon Photonics for Future Systems.

\section{REFERENCES}

1 Lacava, C., Ettabib, M. A., and Petropoulos, P., "Nonlinear Silicon Photonic Signal Processing Devices for Future Optical Networks," Applied Sciences, vol. 7, no. 1, p. 103, 2017.

2 Demas, J., Prabhakar, G., He, T., Ramachandran, S., and Ramachandran, S., "Broadband and Wideband Parametric Gain via Intermodal Four-Wave Mixing in Optical Fiber,' Conference on Lasers and Electro-Optics, no. 2, p. SM3M.1, 2017.

3 Parmigiani, F., Horak, P., Jung, Y., Grüner-Nielsen, L., Geisler, T., Petropoulos, P., and Richardson, D. J., "All-optical mode and wavelength converter based on parametric processes in a three-mode fiber," Optics Express, vol. 25, no. 26, p. 33602, 2017.

4 Essiambre, R., Mestre, M. A., Ryf, R., Gnauck, A. H., Tkach, R. W., Chraplyvy, A. R., Sun, Y., Jiang, X., and Lingle, R., "Experimental Investigation of Inter-Modal Four-Wave Mixing in Few-Mode Fibers," IEEE Photon. Technol. Lett., vol. 25, no. 6, pp. 539-542, 2013.

5 Signorini, S., Mancinelli, M., Bernard, M., Ghulinyan, M., Pucker, G., and Pavesi, L., "Broad wavelength generation and conversion with Multi Modal Four Wave Mixing in sili- con waveguides," in Group IV Photonics, 2017, pp. 59-60. 